\title{
Privacy Preserving Market Basket Data Analysis
}

\author{
Ling Guo, Songtao Guo, and Xintao Wu \\ University of North Carolina at Charlotte, \\ $\{$ lguo2, sguo, xwu $\}$ euncc.edu
}

\begin{abstract}
Randomized Response techniques have been empirically investigated in privacy preserving association rule mining. However, previous research on privacy preserving market basket data analysis was solely focused on support/ confidence framework. Since there are inherent problems with the concept of finding rules based on their support and confidence measures, many other measures (e.g., correlation, lift, etc.) for the general market basket data analysis have been studied. How those measures are affected due to distortion is not clear in the privacy preserving analysis scenario.

In this paper, we investigate the accuracy (in terms of bias and variance of estimates) of estimates of various rules derived from the randomized market basket data and present a general framework which can conduct theoretical analysis on how the randomization process affects the accuracy of various measures adopted in market basket data analysis. We also show several measures (e.g., correlation) have monotonic property, i.e., the values calculated directly from the randomized data are always less or equal than those original ones. Hence, some market basket data analysis tasks can be executed on the randomized data directly without the release of distortion probabilities, which can better protect data privacy.
\end{abstract}

\section{Introduction}

The issue of maintaining privacy in association rule mining has attracted considerable attention in recent years [8[9/421]. Most of techniques are based on a data perturbation or Randomized Response (RR) approach [5], wherein the 0 or 1 ( 0 denotes absence of an item while 1 denotes presence of an item) in the original user transaction vector is distorted in a probabilistic manner that is disclosed to data miners.

However, previous research on privacy preserving market basket data analysis is solely focused on support/confidence framework. In spite of the success of association rules, there are inherent problems with the concept of finding rules based on their support and confidence. Various measures have been studied in market basket data analysis. In this paper we conduct theoretical analysis on the accuracy of various measures adopted previously in market data analysis. Our analysis is based on estimating the parameters of derived random variables. The estimated measure (e.g., Interest statistics) is considered as one derived variable. We present a general method, which is based on the Taylor series, for approximating the mean and variance of derived variables. We also derive interquantile ranges of those estimates. Hence, data miners are ensured that their estimates lie within these ranges with a high confidence.

There exists some scenario where data owners are reluctant to release the distortion probabilities since attackers may exploit those distortion probabilities to recover 
individual data. In this paper, we also show that some useful information can still be discovered directly from the randomized data without those distortion probabilities. Specifically, we show some market basket data analysis tasks (such as correlation analysis or independence hypothetical testing) can be conducted on the randomized data directly without distortion probabilities.

The remainder of this paper is organized as follows. In Section 2, we revisit the distortion framework and discuss how the Randomized Response techniques are applied to privacy preserving association rule mining. In Section 3, we conduct the theoretical analysis on how distortion process affects various other measures adopted in market basket data analysis. In Section 4, we show that some useful data mining results (e.g., dependence itemsets etc.) can be discovered even without the distortion values. We discuss the related work in Section 5 and conclude our work in Section 6.

\section{Distortion Framework Revisited}

The authors in [21:43] proposed the MASK scheme, which is based on Randomized Response, presented strategies of efficiently estimating the original support values of frequent itemsets from the randomized data. Their results empirically shown a high degree of privacy to the user and a high level of accuracy in the mining results can be simultaneously achieved. The privacy situation considered here is that perturbation is done at the level of individual customer records, without being influenced by the contents of the other records in the database. We also focus on a simple independent column perturbation, wherein the value of each attribute in the record is perturbed independently.

\subsection{Randomization Procedure}

Denoting the set of transactions in the database $D$ by $\mathcal{T}=\left\{T_{1}, \cdots, T_{n}\right\}$ and the set of items in the database by $\mathcal{I}=\left\{A_{1}, \cdots, A_{m}\right\}$. Each item is considered as one dichotomous variable with 2 mutually exclusive and exhaustive categories $(0=$ absence, $1=$ presence). Each transaction can be logically considered as a fixed-length sequence of 1 's and 0 's. For item $A_{j}$, we use a $2 \times 2$ distortion probability matrix

$$
P_{j}=\left(\begin{array}{cc}
\theta_{0} & 1-\theta_{0} \\
1-\theta_{1} & \theta_{1}
\end{array}\right)
$$

If the original value is in absence category, it will be kept in absence category with a probability $\theta_{0}$ and changed to presence category with a probability $1-\theta_{0}$. Similarly, if the original value is in presence category, it will be kept in presence with a probability $\theta_{1}$ and changed to absence category with a probability $1-\theta_{1}$. In this paper, we follow the original Warner RR model by simply setting $\theta_{0}=\theta_{1}=p_{j}$.

Let $\pi_{i_{1}, \cdots, i_{k}}$ denote the true proportion corresponding to the categorical combination $\left(A_{1 i_{1}}, \cdots, A_{k i_{k}}\right)$, where $i_{1}, \cdots, i_{k} \in\{0,1\}$. Let $\pi$ be vectors with elements $\pi_{i_{1}, \cdots, i_{k}}$, arranged in a fixed order. The combination vector corresponds to a fixed order of cell entries in the contingency table formed by the $k$-itemset. Table $1(\mathrm{a})$ shows one contingency table for a pair of two variables. We use the notation $\bar{A}(\bar{B})$ to indicate that 
$A(B)$ is absent from a transaction. The vector $\pi=\left(\pi_{00}, \pi_{01}, \pi_{10}, \pi_{11}\right)^{\prime}$ corresponds to a fixed order of cell entries $\pi_{i j}$ in the $2 \times 2$ contingency table. $\pi_{11}$ denotes the proportion of transactions which contain both $A$ and $B$ while $\pi_{10}$ denotes the proportion of transactions which contain $A$ but not $B$. The row sum $\pi_{1+}$ represents the support frequency of item $A$ while the column sum $\pi_{+1}$ represents the support frequency of item $B$.

The original database $D$ is changed to $D_{\text {ran }}$ after randomization. Assume $\lambda_{\mu_{1}, \cdots, \mu_{k}}$ is the probability of getting a response $\left(\mu_{1}, \cdots, \mu_{k}\right)$ and $\lambda$ the vector with elements $\lambda_{\mu_{1}, \cdots, \mu_{k}}$ arranged in a fixed order(e.g., the vector $\lambda=\left(\lambda_{00}, \lambda_{01}, \lambda_{10}, \lambda_{11}\right)^{\prime}$ corresponds to cell entries $\lambda_{i j}$ in the randomized contingency table as shown in Table 1(b), we can get

$$
\lambda=\left(P_{1} \times \cdots \times P_{k}\right) \pi
$$

where $\times$ stands for the Kronecker product. Let $P=P_{1} \times \cdots \times P_{k}$, an unbiased estimate of $\pi$ follows as

$$
\hat{\pi}=P^{-1} \hat{\lambda}=\left(P_{1}^{-1} \times \cdots \times P_{k}^{-1}\right) \hat{\lambda}
$$

where $\hat{\lambda}$ is the vector of proportions observed from the randomized data corresponding to $\lambda$ and $P_{j}^{-1}$ denotes the inverse of the matrix $P_{j}$. Note that although the distortion matrices $P_{1}, \cdots, P_{k}$ are known, they can only be utilized to estimate the proportions of itemsets of the original data, rather than the precise reconstruction of the original $0-1$ data.

\subsection{Accuracy of Association Rule}

Recently the authors in [11] investigated the accuracy of support and confidence measures for each individual association rule derived from the randomized data and presented an analytical formula for evaluating their accuracy in terms of bias and variance. From the derived variances, users can tell how accurate the derived association rules in terms of both support and confidence measures from the randomized data are.

Table 1. $2 \times 2$ contingency tables for two variables A,B

(a) Original

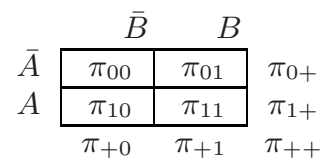

(b) After randomization

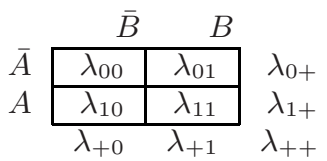

Assume item $A$ and $B$ are randomized using distortion matrix $P_{1}$ and $P_{2}$ respectively. For a simple association rule $A \Rightarrow B$ derived from the randomized data, it was shown in [11] that an unbiased estimate is $\hat{\pi}=P^{-1} \hat{\lambda}=\left(P_{1}^{-1} \times P_{2}^{-1}\right) \hat{\lambda}$ with the covariance matrix as

$$
\operatorname{cov} v(\hat{\pi})=(n-1)^{-1} P^{-1}\left(\hat{\lambda}^{\delta}-\hat{\lambda} \hat{\lambda}^{\prime}\right) P^{\prime-1}
$$

where $\hat{\lambda}^{\delta}$ is a diagonal matrix with the same diagonal elements as those of $\hat{\lambda}$ arranged in the same order. The last element of $\hat{\pi}$ corresponds to the estimated support value $s$ 
and the last element of $\operatorname{cov} v(\hat{\pi})$ denotes its estimated variance. The estimated confidence $c$ is

$$
\hat{c}=\frac{\hat{s}_{A B}}{\hat{s}_{A}}=\frac{\hat{\pi}_{11}}{\hat{\pi}_{1+}}
$$

and its variance as

$$
\hat{v} \operatorname{ar}(\hat{c}) \approx \frac{\hat{\pi}_{10}^{2}}{\hat{\pi}_{1+}^{4}} \hat{\operatorname{var}}\left(\hat{\pi}_{11}\right)+\frac{\hat{\pi}_{11}^{2}}{\hat{\pi}_{1+}^{4}} \hat{\operatorname{var}}\left(\hat{\pi}_{10}\right)-2 \frac{\hat{\pi}_{10} \hat{\pi}_{11}}{\hat{\pi}_{1+}^{4}} \operatorname{cov} v\left(\hat{\pi}_{11}, \hat{\pi}_{10}\right)
$$

The above results can be straightforwardly extended to the general association rule $\mathcal{X} \Rightarrow \mathcal{Y}$. Incorporating the derived estimate and variance, the $(1-\alpha) 100 \%$ interquantile range for the estimated support and confidence is then derived. An $(1-\alpha) 100 \%$ interquantile range, say $\alpha=0.05$, shows the interval where the original value lies in with $95 \%$ probability. In other words, users shall have $95 \%$ confidence that the original value falls into this interquantile range.

\section{Accuracy Analysis of Measures}

The objective interestingness measure is usually computed from the contingency table. Table 2 shows various measures defined for a pair of binary variables [23]. Here we give results on how RR may affect the accuracy of those measures or analysis methods on market basket data.

\begin{tabular}{|c|c|c|c|}
\hline Measure & Expression & Measure & Expression \\
\hline Support $(s)$ & $\pi_{11}$ & Confidence $(c)$ & $\frac{\pi_{11}}{\pi_{1+}}$ \\
\hline Correlation $(\phi)$ & $\frac{\pi_{11} \pi_{00}-\pi_{01} \pi_{10}}{\sqrt{\pi_{1+} \pi_{1} \pi_{0+} \pi_{+0}}}$ & Cosine (IS) & $\frac{\pi_{11}}{\sqrt{\pi_{1+\pi_{+1}}}}$ \\
\hline Odds ratio $(\alpha)$ & $\frac{\pi_{11} \pi_{00}}{\pi_{10} \pi 01}$ & Interest (I) & $\frac{\pi_{11}}{\pi_{1+} \pi_{+1}}$ \\
\hline Jaccard $(\zeta)$ & $\frac{\pi_{11}}{\pi_{1+}+\pi_{+1}-\pi_{11}}$ & (PS) & $\pi_{11}-\pi_{1+} \pi_{+1}$ \\
\hline Mutual Info(M) & $\frac{\sum_{i} \sum_{j} \pi_{i j} \log \frac{\pi_{i j}}{\pi_{i+} \pi_{+j}}}{-\sum_{i} \pi_{i+} \log \pi_{i+}}$ & Conviction (V) & $\frac{\pi_{1+} \pi_{+0}}{\pi_{10}}$ \\
\hline $\mathrm{J}$-measure $(\mathrm{J})$ & $\pi_{11} \log \frac{\pi_{11}}{\pi_{1+} \pi_{+1}}+\pi_{10} \log \frac{\pi_{11}}{\pi_{1+} \pi_{+0}}$ & Certainty $(\mathrm{F})$ & $\frac{\frac{\pi_{11}}{\pi_{1+}}-\pi_{1+}}{1-\pi_{+1}}$ \\
\hline Std. residues $(\mathrm{e})$ & $\frac{\pi_{i j}-\pi_{i+\pi_{+j}}}{\sqrt{\pi_{i+}+\pi_{+j}}}$ & Likelihood $\left(G^{2}\right)$ & $2 \sum_{i} \sum_{j} \pi_{i j} \log \frac{\pi_{i j}}{\pi_{i+} \pi_{+j}}$ \\
\hline Pearson $\left(\chi^{2}\right)$ & $\sum_{i} \sum_{j} \frac{\left\{\pi_{i j}-\pi_{i+} \pi_{+j}\right\}^{2}}{\pi_{i+} \pi_{+j}}$ & Added Value(AV) & $\frac{\pi_{11}}{\pi_{1+}-\pi_{+1}}$ \\
\hline
\end{tabular}

Table 2. Objective measures for the itemset $\{A, B\}$

In this Section, we provide a general framework which can derive estimates of all measures using randomized data and the released distortion parameters. Furthermore, we present a general approach which can calculate the variance of those estimates in Section 3.1. By incorporating the Chebyshev Theorem, we show how to derive their interquantile ranges in Section 3.2. 


\subsection{Variances of Derived Measures}

From Table 2, we can see that each measure can be expressed as one derived random variable (or function) from the observed variables $\left(\pi_{i j}\right.$ or their marginal totals $\left.\pi_{i+}, \pi_{+j}\right)$. Similarly, its estimate from the randomized data can be considered as another derived random variable from the input variables $\left(\hat{\pi}_{i j}, \hat{\pi}_{i+}, \hat{\pi}_{+j}\right)$. Since we know how to derive variances of the input variables $\left(\hat{v} a r\left(\hat{\pi}_{i j}\right)\right)$ from the randomized data, our problem is then how to derive the variance of the derived output variable.

In the following, we first present a general approach based on the delta method [17] and then discuss how to derive the variance of chi-square statistics $\left(\chi^{2}\right)$ as one example.

Let $z$ be a random variable derived from the observed random variables $x_{i}(i=$ $1, \cdots, n): z=g(x)$. According to the delta method, a Taylor approximation of the variance of a function with multiple variables can be expanded as

$$
\operatorname{var}\{g(x)\}=\sum_{i=1}^{k}\left\{g_{i}^{\prime}(\theta)\right\}^{2} \operatorname{var}\left(x_{i}\right)+\sum_{i \neq j=1}^{k} \sum_{i} g_{i}^{\prime}(\theta) g_{j}^{\prime}(\theta) \operatorname{cov}\left(x_{i}, x_{j}\right)+o\left(n^{-r}\right)
$$

where $\theta_{i}$ is the mean of $x_{i}, g(x)$ stands for the function $g\left(x_{1}, x_{2}, \cdots, x_{k}\right), g_{i}^{\prime}(\theta)$ is the $\frac{\partial g(x)}{\partial x_{i}}$ evaluated at $\theta_{1}, \theta_{2}, \cdots, \theta_{k}$.

For market basket data with 2 variables, $\hat{\pi}=\left(\hat{\pi}_{00}, \hat{\pi}_{01}, \hat{\pi}_{10}, \hat{\pi}_{11}\right)^{\prime}$, the estimated chi-square is shown as

$$
\begin{aligned}
\hat{\chi}^{2} & =n\left(\frac{\left(\hat{\pi}_{00}-\hat{\pi}_{0+} \hat{\pi}_{+0}\right)^{2}}{\hat{\pi}_{0+} \hat{\pi}_{+0}}+\frac{\left(\hat{\pi}_{01}-\hat{\pi}_{0+} \hat{\pi}_{+1}\right)^{2}}{\hat{\pi}_{0+} \hat{\pi}_{+1}}\right. \\
& \left.+\frac{\left(\hat{\pi}_{10}-\hat{\pi}_{1+} \hat{\pi}_{+0}\right)^{2}}{\hat{\pi}_{1+} \hat{\pi}_{+0}}+\frac{\left(\hat{\pi}_{11}-\hat{\pi}_{1+} \hat{\pi}_{+1}\right)^{2}}{\hat{\pi}_{1+} \hat{\pi}_{+1}}\right)
\end{aligned}
$$

Let $x_{1}=\hat{\pi}_{00}, x_{2}=\hat{\pi}_{01}, x_{3}=\hat{\pi}_{10}$ and $x_{4}=\hat{\pi}_{11}$, we have

$$
\begin{aligned}
& g\left(x_{1}, x_{2}, x_{3}, x_{4}\right)=\chi^{2} \\
& =n\left[\frac{x_{1}^{2}}{\left(x_{1}+x_{2}\right)\left(x_{1}+x_{3}\right)}+\frac{x_{2}^{2}}{\left(x_{1}+x_{2}\right)\left(x_{2}+x_{4}\right)}+\right. \\
& \left.\frac{x_{3}^{2}}{\left(x_{3}+x_{4}\right)\left(x_{3}+x_{1}\right)}+\frac{x_{4}^{2}}{\left(x_{4}+x_{3}\right)\left(x_{4}+x_{2}\right)}-1\right]
\end{aligned}
$$

Partial derivatives of the function $g()$ can be calculated respectively. By incorporating estimated expectations, variances and covariances of variables in function $g()$, the variance of function $g()$ can be estimated as

$$
\begin{aligned}
& \hat{v} \operatorname{ar}(g) \approx G_{1}^{2} \hat{v} \operatorname{ar}\left(\hat{\pi}_{00}\right)+G_{2}^{2} \hat{v} \operatorname{ar}\left(\hat{\pi}_{01}\right)+G_{3}^{2} \hat{\operatorname{var}}\left(\hat{\pi}_{10}\right)+G_{4}^{2} \hat{\operatorname{var}}\left(\hat{\pi}_{11}\right) \\
& +2 G_{1} G_{2} \hat{\operatorname{cov}}\left(\hat{\pi}_{00}, \hat{\pi}_{01}\right)+2 G_{1} G_{3} \hat{\operatorname{cov}}\left(\hat{\pi}_{00}, \hat{\pi}_{10}\right)+2 G_{1} G_{4} \hat{\operatorname{cov}}\left(\hat{\pi}_{00}, \hat{\pi}_{11}\right) \\
& +2 G_{2} G_{3} \hat{\operatorname{cov}}\left(\hat{\pi}_{01}, \hat{\pi}_{10}\right)+2 G_{2} G_{4} \hat{\operatorname{cov}}\left(\hat{\pi}_{01}, \hat{\pi}_{11}\right)+2 G_{3} G_{4} \hat{\operatorname{cov}}\left(\hat{\pi}_{10}, \hat{\pi}_{11}\right)
\end{aligned}
$$


where

$$
\begin{aligned}
& G_{1}=\frac{\partial g}{\partial x_{1}}=n\left[\frac{\hat{\pi}_{00}^{2}\left(\hat{\pi}_{01}+\hat{\pi}_{10}\right)+2 \hat{\pi}_{00} \hat{\pi}_{01} \hat{\pi}_{10}}{\hat{\pi}_{0+}^{2} \hat{\pi}_{+0}^{2}}-\frac{\hat{\pi}_{01}^{2}}{\hat{\pi}_{0+}^{2} \hat{\pi}_{+1}}-\frac{\hat{\pi}_{10}^{2}}{\hat{\pi}_{+0}^{2} \hat{\pi}_{1+}}\right] \\
& G_{2}=\frac{\partial g}{\partial x_{2}}=n\left[\frac{\hat{\pi}_{01}^{2}\left(\hat{\pi}_{00}+\hat{\pi}_{11}\right)+2 \hat{\pi}_{00} \hat{\pi}_{01} \hat{\pi}_{11}}{\hat{\pi}_{0+}^{2} \hat{\pi}_{+1}^{2}}-\frac{\hat{\pi}_{00}^{2}}{\hat{\pi}_{0+}^{2} \hat{\pi}_{+0}}-\frac{\hat{\pi}_{11}^{2}}{\hat{\pi}_{+1}^{2} \hat{\pi}_{1+}}\right] \\
& G_{3}=\frac{\partial g}{\partial x_{3}}=n\left[\frac{\hat{\pi}_{10}^{2}\left(\hat{\pi}_{11}+\hat{\pi}_{00}\right)+2 \hat{\pi}_{00} \hat{\pi}_{10} \hat{\pi}_{11}}{\hat{\pi}_{1+}^{2} \hat{\pi}_{+0}^{2}}-\frac{\hat{\pi}_{11}^{2}}{\hat{\pi}_{1+}^{2} \hat{\pi}_{+1}}-\frac{\hat{\pi}_{00}^{2}}{\hat{\pi}_{+0}^{2} \hat{\pi}_{0+}}\right] \\
& G_{4}=\frac{\partial g}{\partial x_{4}}=n\left[\frac{\hat{\pi}_{11}^{2}\left(\hat{\pi}_{01}+\hat{\pi}_{10}\right)+2 \hat{\pi}_{11} \hat{\pi}_{01} \hat{\pi}_{10}}{\hat{\pi}_{1+}^{2} \hat{\pi}_{+1}^{2}}-\frac{\hat{\pi}_{10}^{2}}{\hat{\pi}_{1+}^{2} \hat{\pi}_{+0}}-\frac{\hat{\pi}_{01}^{2}}{\hat{\pi}_{+1}^{2} \hat{\pi}_{0+}}\right]
\end{aligned}
$$

Since $\chi^{2}=n \phi^{2}$ where $\phi$ denotes correlation (A proof is given in Appendix A of [22]), $\phi=\sqrt{\chi^{2} / n}=\sqrt{g / n}$. As we know, $\frac{\partial \phi}{\partial x_{i}}=\frac{1}{2 \sqrt{g n}} \frac{\partial g}{\partial x_{i}}$. Following the same procedure above, the variance of correlation $\phi$ can be approximated as

$$
\hat{v} a r(\phi) \approx \frac{\hat{v} a r(g)}{4 G_{E}}
$$

where

$$
G_{E}=n^{2}\left[\frac{\hat{\pi}_{00}^{2}}{\hat{\pi}_{0+} \hat{\pi}_{+0}}+\frac{\hat{\pi}_{01}^{2}}{\hat{\pi}_{0+} \hat{\pi}_{+1}}+\frac{\hat{\pi}_{10}^{2}}{\hat{\pi}_{1+} \hat{\pi}_{+0}}+\frac{\hat{\pi}_{11}^{2}}{\hat{\pi}_{1+} \hat{\pi}_{+1}}-1\right] .
$$

Similarly we can derive variances of the estimated values of all measures shown in Table 2. Measures such as $\chi^{2}$, interest factor, IS, PS, and Jaccard coefficient can be extended to more than two variables using the multi-dimensional contingency tables. We show the estimated chi-square statistics for $k$-itemset as one example.

$$
\hat{\chi}^{2}=n \sum_{u_{1}=0}^{1} \cdots \sum_{u_{k}=0}^{1} \frac{\left(\hat{\pi}_{u_{1} \cdots u_{k}}-\prod_{j=1}^{k} \hat{\pi}_{u_{j}}^{(j)}\right)^{2}}{\prod_{j=1}^{k} \hat{\pi}_{u_{j}}^{(j)}}
$$

It is easy to see $\hat{\chi}^{2}$ can be considered as one derived variable from the observed elements $\hat{\pi}_{u_{1} \cdots u_{k}}$ and the marginal totals $\hat{\pi}_{u_{j}}^{(j)}$ of the $2^{k}$ contingency table. Following the same delta method, we can derive its variance.

\subsection{Interquantile Ranges of Derived Measures}

To derive interquantile ranges of estimates, we need to explore the distribution of those derived variables. In [11], the authors have shown the estimate of support follows an approximate normal distribution and the estimate of confidence (i.e., a ratio of two correlated normal variables) follows a very complex $F(w)$ distribution. In general, we can observe that every element (e.g., $\hat{\pi}_{i j}$ ) in the derived measure expressions (shown in Table 2) has an approximate normal distribution, however, the derived measures usually do not have explicit distribution expressions. Hence we cannot calculate the critical values of distributions to derive the interquantile range. In the following, we provide an approximation to such range based on Chebyshev's theorem. 
Theorem 1. (Chebyshev's theorem) Let $X$ be a random variable with expected value $\mu$ and finite variance $\sigma^{2}$. Then for any real $k>0$, we have $\operatorname{Pr}(|X-\mu| \geq k \sigma) \leq 1 / k^{2}$.

Chebyshev's Theorem gives a conservative estimate. It provides a lower bound to the proportion of measurements that are within a certain number of standard deviations from the mean. The theorem can be useful despite loose bounds because it can be applied to random variables of any distribution, and because these bounds can be calculated knowing no more about the distribution than the mean and variance. For example, the loose $(1-\alpha) 100 \%$ interquantile range of correlation $\phi$ between $A$ and $B$ can be approximated as

$$
\left[\hat{\phi}-\frac{1}{\sqrt{\alpha}} \sqrt{\hat{v} \operatorname{ar}(\hat{\phi})}, \hat{\phi}+\frac{1}{\sqrt{\alpha}} \sqrt{\hat{\operatorname{var}(\hat{\phi})}}\right]
$$

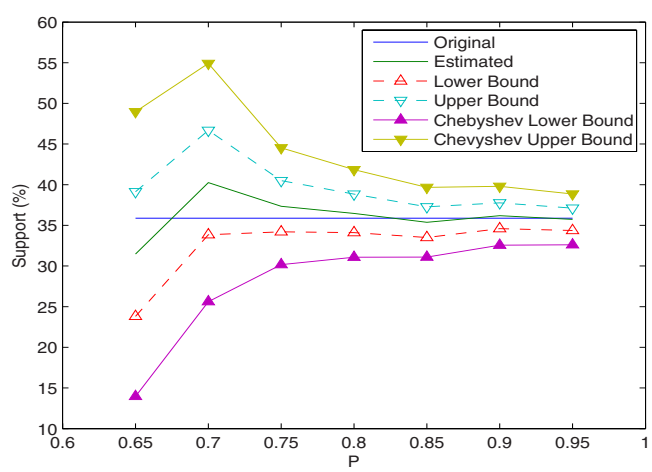

Fig. 1. Interquantile Range vs. varying $p$

From Chebyshev's theorem, we know for any sample, at least $\left(1-(1 / k)^{2}\right)$ of the observations in the data set fall within $k$ standard deviations of the mean. When we set $\alpha=\frac{1}{k^{2}}$, we have $\operatorname{Pr}\left(|X-\mu| \geq \frac{1}{\sqrt{\alpha}} \sigma\right) \leq \alpha$. Hence, $\operatorname{Pr}\left(|X-\mu| \leq \frac{1}{\sqrt{\alpha}} \sigma\right) \geq 1-\alpha$. The $(1-\alpha) 100 \%$ interquantile range of the estimated measure is then derived.

Note that the interquantile range based on Chebyshev's Theorem is much larger than that based on known distributions such as normal distribution for support estimates. This is because that $\frac{1}{\sqrt{\alpha}} \geq z_{\alpha / 2}$ where $z_{\alpha / 2}$ is the upper $\alpha / 2$ critical value for the standard normal distribution. In Figure 1, we show how the $95 \%$ interquantile ranges for the estimated support of one particular rule ( $G \Rightarrow H$ from COIL data) change with varied distortion $p$ from 0.65 to 0.95 . We can see the interquantile range derived based on Chebyshev's theorem is wider than that derived from known normal distribution. As expected, we can also observe that the larger the $p$, the more accurate the estimate and the tighter the interquantile ranges.

\section{Measures Derived from the Randomized Data Without $p$}

Randomization still runs certain risk of disclosures. Attackers may exploit the released distortion parameter $p$ to calculate the posterior probabilities of the original value based 
on the distorted data. It is considered as jeopardizing with respect to the original value if the posterior probabilities are significantly greater than the a-priori probabilities [5]. In this section, we consider the scenario where the distortion parameter $p$ is not released to data miners. As a result individual privacy can be better preserved.

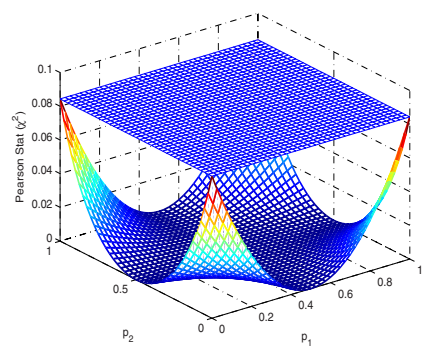

(a) $\chi^{2}$ Statistics

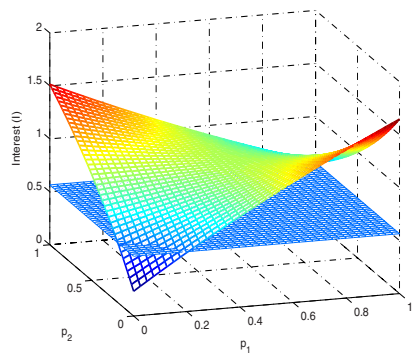

(b) Interest

Fig. 2. measures from randomized data vs. varying $p_{1}$ and $p_{2}$

Result 1. For any pair of items $A, B$ perturbed with distortion parameter $p_{1}$ and $p_{2}$ $\left(p_{1}, p_{2} \in[0,1]\right)$ respectively, we have

$$
\begin{aligned}
\phi_{\text {ran }} & \leq \phi_{\text {ori }} & M_{\text {ran }} & \leq M_{\text {ori }} \\
G_{\text {ran }}^{2} & \leq G_{\text {ori }}^{2} & \chi_{\text {ran }}^{2} & \leq \chi_{\text {ori }}^{2}
\end{aligned}
$$

where $\phi_{\text {ori }}, M_{\text {ori }}, G_{\text {ori }}^{2}, \chi_{\text {ori }}^{2}$ denote Correlation, Mutual Information, Likelihood Ratio, Pearson Statistics measures calculated from the original data respectively and $\phi_{\text {ran }}, M_{\text {ran }}, G_{\text {ran }}^{2}, \chi_{\text {ran }}^{2}$ correspond to measures calculated directly from the randomized data without knowing $p_{1}$ and $p_{2}$.

All other measures shown in Table 2 do not hold monotonic relations.

Proof. we include the proof of $\chi^{2}$ in Appendix A and we skip proof of all other measures due to space limits.

Figure 2(a) and 2(b) show how the $\chi^{2}$ Statistics $(G$ and $H$ ) and Interest measures calculated from the randomized data varies with distortion parameters $p_{1}$ and $p_{2}$. We can easily observe that $\chi_{\text {ran }}^{2} \leq \chi_{\text {ori }}^{2}$ for all $p_{1}, p_{2} \in[0,1]$ and $I_{\text {ran }} \geq I_{\text {ori }}$ for some $p_{1}, p_{2}$ values.

We would emphasize that Result 1 is important for data exploration tasks such as hypothesis testing. It shows useful information can still be discovered from the randomized data even without knowing the distortion parameters. For example, testing pairwise independence between the original attributes is equivalent to testing pairwise independence between the corresponding distorted attributes. From the randomized data, if we discover an itemset which satisfies $\chi_{\text {ran }}^{2} \geq \chi_{\alpha}^{2}$, we can guarantee that dependence exists among the original itemset since $\chi_{\text {ran }}^{2} \leq \chi_{\text {ori }}^{2}$ holds for all $p 1$.

\footnotetext{
${ }^{1}$ The alternative hypothesis will be accepted if the observed data values are sufficiently improbable under the null hypothesis. Otherwise, the null hypothesis is not rejected.
} 


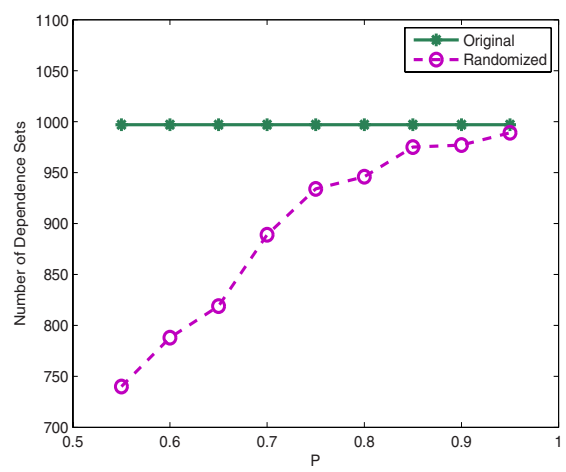

Fig. 3. The number of dependence itemsets vs. varying $p$

Figure 3 shows the number of dependence itemsets discovered from the randomized COIL data sets with varying $p$ from 0.55 to 0.95 . We can observe that the larger the distortion parameter $p$, the more dependence itemsets calculated directly from the randomized data. Even with $p=0.55$, around 750 dependence sets can be discovered from the randomized data, which represents about $75 \%$ of 997 dependence itemsets derived from the original data.

\section{Related Work}

Privacy is becoming an increasingly important issue in many data mining applications. A considerable amount of work on privacy preserving data mining, such as additive randomization based [21] and projection based [6]19], has been proposed. Recently, a lot of research has focused on the privacy aspect of the above approaches and various point-wise reconstruction methods [16 15 |13 $|12| 18 \mid 14]$ have been investigated.

The issue of maintaining privacy in association rule mining has also attracted con-

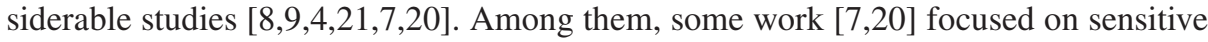
association rule hiding where privacy is defined in terms of the output frequent itemsets or association rules. The work closest to our approach is that of [214]3] based on Randomization Response techniques. In [214], the authors proposed the MASK technique to preserve privacy for frequent itemset mining. In [4], the authors addressed the issue of providing efficiency in estimating support values of itemsets derived from the randomized data. Our paper focused on the issue of providing accuracy in terms of various reconstructed measures (e.g., support, confidence, correlation, lift, etc.) in privacy preserving market basket data analysis. Providing the accuracy of discovered patterns from randomized data is important for data miners. To the best of our knowledge, this has not been previously explored in the context of privacy preserving data mining although defining the significance of discovered patterns in general data mining has been studied (e.g., [10]). 


\section{Conclusion}

In this paper, we have considered the issue of providing accuracy in privacy preserving market basket data analysis. We have presented a general approach to deriving variances of estimates of various measures adopted in market basket data analysis. We applied the idea of using interquantile ranges based on Chebyshev's Theorem to bound those estimates derived from the randomized market basket data. We theoretically show some measures (e.g., correlation) have monotonic property, i.e., the measure values calculated directly from the randomized data are always less than or equal to those original ones. As a result, there is no risk to introduce false positive patterns. Hence, some market basket data analysis tasks (such as correlation analysis or independence hypothetical testing) can be executed on the randomized data directly without the release of distortion probabilities. In the future, we are interested in exploring the tradeoff between the privacy of individual data and the accuracy of data mining results. We will also investigate how various measures are affected by randomization, e.g., which measures are more sensible to randomization.

\section{Acknowledgments}

This work was supported in part by U.S. National Science Foundation IIS-0546027.

\section{References}

1. Agrawal, D., Agrawal, C.: On the design and quantification of privacy preserving data mining algorithms. In: Proceedings of the 20th Symposium on Principles of Database Systems (2001)

2. Agrawal, R., Srikant, R.: Privacy-preserving data mining. In: Proceedings of the ACM SIGMOD International Conference on Management of Data, pp. 439-450. Dallas, Texas (May 2000)

3. Agrawal, S., Haritsa, J.: A framework for high-accuracy privacy-preserving mining. In: Proceedings of the 21st IEEE International Conference on Data Engineering, pp. 193-204. IEEE Computer Society Press, Los Alamitos (2005)

4. Agrawal, S., Krishnan, V., Haritsa, J.: On addressing efficiency concerns in privacypreserving mining. Proc. of 9th Intl. Conf. on Database Systems for Advanced Applications (DASFAA), pp. 113-124 (2004)

5. Chaudhuri, A., Mukerjee, R.: Randomized Response Theory and Techniques. Marcel Dekker (1988)

6. Chen, K., Liu, L.: Privacy preserving data classification with rotation perturbation. In: Proceedings of the 5th IEEE International Conference on Data Mining. Houston,TX (November 2005)

7. Dasseni, E., Verykios, V., Elmagarmid, A.K., Bertino, E.: Hiding association rules by using confidence and support. In: Proceedings of the 4th International Information Hiding Workshop, pp. 369-383. Pittsburg, PA (April 2001)

8. Evfimievski, A., Gehrke, J., Srikant, R.: Limiting privacy breaches in privacy preserving data mining. In: Proceedings of the twenty-second ACM SIGMOD-SIGACT-SIGART symposium on Principles of database systems, pp. 211-222. ACM Press, New York (2003) 
9. Evfimievski, A., Srikant, R., Agrawal, R., Gehrke, J.: Privacy preserving mining of association rules. In: Proceedings of the eighth ACM SIGKDD international conference on Knowledge discovery and data mining, pp. 217-228 (2002)

10. Gionis, A., Mannila, H., Mielikainen, T., Tsaparas, P.: Assessing data mining results via swap randomization. In: Proceedings of the 12th ACM International Conference on Knowledge Discovery and Data Mining (2006)

11. Guo, L., Guo, S., Wu, X.: On addressing accuracy concerns in privacy preserving association rule mining. Technical Report, CS Dept. UNC Charlotte (March 2007)

12. Guo, S., Wu, X.: On the lower bound of reconstruction error for spectral filtering based privacy preserving data mining. In: Proceedings of the 10th European Conference on Principles and Practice of Knowledge Discovery in Databases, pp. 520-527. Berlin, Germany (September 2006)

13. Guo, S., Wu, X.: On the use of spectral filtering for privacy preserving data mining. In: Proceedings of the 21st ACM Symposium on Applied Computing, pp. 622-626 (April 2006)

14. Guo, S., Wu, X.: Deriving private information from arbitraraily projected data. In: Proceedings of the 11th Pacific-Asia Conference on Knowledge Discovery and Data Mining, pp. 84-95. Nanjing, China, (May 2007)

15. Huang, Z., Du, W., Chen, B.: Deriving private information from randomized data. In: Proceedings of the ACM SIGMOD Conference on Management of Data, Baltimore, MA (2005)

16. Kargupta, H., Datta, S., Wang, Q., Sivakumar, K.: On the privacy preserving properties of random data perturbation techniques. In: Proceedings of the $3 \mathrm{rd}$ International Conference on Data Mining, pp. 99-106 (2003)

17. Kendall, M.G., Stuart, A.: The advanced theory of statistics. Hafner Pub. Co, New York (1969)

18. Liu, K., Giannella, C., Kargupta, H.: An attacker's view of distance preserving maps for privacy preserving data mining. In: Proceedings of the 10th European Conference on Principles and Practice of Knowledge Discovery in Databases. Berlin, Germany (September 2006)

19. Liu, K., Kargupta, H., Ryan, J.: Random projection based multiplicative data perturbation for privacy preserving distributed data mining. IEEE Transaction on Knowledge and Data Engineering 18(1), 92-106 (2006)

20. Oliveira, S., Zaiane, O.: Protecting sensitive knowledge by data sanitization. In: Proceedings of the 3rd IEEE International Conference on Data Mining, pp. 211-218. Melbourne, Florida (November 2003)

21. Rizvi, S., Haritsa, J.: Maintaining data privacy in association rule mining. In: Proceedings of the 28th International Conference on Very Large Data Bases (2002)

22. Silverstein, C., Brin, S., Motwani, R., Ullman, J.: Scalable techniques for mining causal structures. In: Proceedings of the 24th VLDB Conference. New York (1998)

23. Tan, P., Steinbach, M., Kumar, K.: Introuduction to Data Mining. Addison-Wesley, Reading (2006)

\section{A Proof of Result 1}

The chi-square calculated directly from the randomized data without knowing $\mathrm{p}$ is

$$
\chi_{\text {ran }}^{2}=\frac{n\left(\lambda_{11}-\lambda_{1+} \lambda_{+1}\right)^{2}}{\lambda_{1+} \lambda_{+1} \lambda_{0+} \lambda_{+0}}
$$


The original chi-square can be expressed as

$$
\begin{aligned}
\chi_{\text {ori }}^{2} & =\frac{n\left(\pi_{11}-\pi_{1+} \pi_{+1}\right)^{2}}{\pi_{1+} \pi_{+1} \pi_{0+} \pi_{+0}} \\
& =\frac{n\left(\lambda_{11}-\lambda_{1+} \lambda_{+1}\right)^{2}}{f\left(p_{1}, p_{2}, \lambda_{0+}, \lambda_{1+}, \lambda_{+0}, \lambda_{+1}\right)+\lambda_{1+} \lambda_{+1} \lambda_{0+} \lambda_{+0}}
\end{aligned}
$$

where $f\left(p_{1}, p_{2}, \lambda_{0+}, \lambda_{1+}, \lambda_{+0}, \lambda_{+1}\right)=$ $p_{1} p_{2}\left(p_{1}-1\right)\left(p_{2}-1\right)+p_{1}\left(p_{1}-1\right) \lambda_{+0} \lambda_{+1}+p_{2}\left(p_{2}-1\right) \lambda_{0+} \lambda_{1+}$

To prove $\chi_{\text {ran }}^{2} \leq \chi_{\text {ori }}^{2}$, we need $f\left(p_{1}, p_{2}, \lambda_{0+}, \lambda_{1+}, \lambda_{+0}, \lambda_{+1}\right) \leq 0$ holds for $\forall$ $\left\{p_{1}, p_{2}, \lambda_{0+}, \lambda_{1+}, \lambda_{+0}, \lambda_{+1}\right\}$.

As

$$
\begin{array}{ll}
\lambda_{+0}=\pi_{+0} p_{2}+\left(1-\pi_{+0}\right)\left(1-p_{2}\right) & \lambda_{+1}=1-\lambda_{+0} \\
\lambda_{0+}=\pi_{0+} p_{1}+\left(1-\pi_{0+}\right)\left(1-p_{1}\right) & \lambda_{1+}=1-\lambda_{0+}
\end{array}
$$

$f()$ can be expressed as a function with parameters $p_{1}$ and $p_{2}$.

We can prove $f() \leq 0 \forall p_{1}, p_{2} \in[1 / 2,1]$ by showing 1$) f()$ is monotonically increasing with $p_{1}$ and $p_{2}$ and 2) $f\left(p_{1}=1, p_{2}=1\right)=0$.

1) Since $p_{1}$ and $p_{2}$ are symmetric and independent, $f()$ can be expressed as

$$
\begin{aligned}
f\left(p_{1}\right) & =a p_{1}^{2}-a p_{1}+\pi_{0+}\left(1-\pi_{0+}\right) \\
a & =m p_{2}^{2}-m p_{2}+\left(1-\pi_{+0}\right) \pi_{+0} \\
m & =-4 \pi_{+0}^{2}+4 \pi_{+0}-4 \pi_{0+}^{2}+4 \pi_{0+}-1
\end{aligned}
$$

Note that $f\left(p_{1}\right)$ is monotonically increasing if $a \geq 0$. Since $0 \leq \pi_{+0}, \pi_{0+} \leq 1$, we have $-1 \leq m \leq 1$.

- When $0 \leq m \leq 1$,

$$
\Delta=m^{2}-4 m\left(1-\pi_{+0}\right) \pi_{+0}=-m\left(2 \pi_{0+}-1\right)^{2} \leq 0
$$

we have $a \geq 0$.

- When $-1 \leq m<0$, since $\Delta=m^{2}-4 m\left(1-\pi_{+0}\right) \pi_{+0} \geq m^{2}>0$ the roots for Equation 6 are $p_{21}=\frac{m-\sqrt{\Delta}}{2 m}>1$ and $p_{22}=\frac{m+\sqrt{\Delta}}{2 m}<0$, hence we have $a \geq 0$ for all $1 / 2 \leq p_{2} \leq 1$

Since $a \geq 0$, we have proved $f()$ is monotonically increasing with $p_{1}$. Similarly, we can prove $f()$ is monotonically increasing with $p_{2}$.

2) It is easy to check $f\left(p_{1}, p_{2}\right)=0$ when $p_{1}=p_{2}=1$.

Combining 1) and 2), we have proved $f() \leq 0 \forall p_{1}, p_{2} \in[1 / 2,1]$. Hence, we have $\chi_{\text {ran }}^{2} \leq \chi_{\text {ori }}^{2}$. 